\title{
LARGE TIME BEHAVIOR OF SOLUTIONS FOR THE GENERALIZED KADOMTSEV-PETVIASHVILI EQUATION
}

\section{TOMOYUKI NiIZATO}

Abstract. We consider the Cauchy problem for the generalized Kadomtsev-Petviashvili (KP) equation

$$
\begin{cases}u_{t}+u_{x x x}+\sigma \partial_{x}^{-1} u_{y y}=-\left(u^{\rho}\right)_{x}, & (x, y) \in \mathbb{R}^{2}, t \in \mathbb{R}, \\ u(0, x, y)=u_{0}(x, y), & (x, y) \in \mathbb{R}^{2},\end{cases}
$$

where $\sigma=1$ or $\sigma=-1, \partial_{x}^{-1}=\int_{-\infty}^{x} d x^{\prime}$. Hayashi-Naumkin-Saut [2] have shown asymptotics of solutions for KP equation when $\rho \geqslant 3$ and the initial data is sufficiently small and regular. Our aim is to fill the gap of the proof of $\mathbf{L}^{\infty}$ time decay of small solutions obtained in [2] and improve their result on the regularity of the data.

Mathematics subject classification (2010): 35Q53.

Keywords and phrases: Kadomtsev-Petviashvili equation, scattering problem.

\section{REFERENCES}

[1] A. Friedman, Partial Differential Equations. New York: Holt-Rinehart and Winston, 1969.

[2] N. HaYAShi, P. I. NAUm KIN, AND J. C. SAUT, Asymptotics for large time of global solutions to the generalized Kadomtsev-Petviashvili Equation, Commun. Math. Phys., 201 (1999), 577-590.

[3] P. Isaza, J. Mejia And V. Stallbohm, Local solution for the Kadomtsev-Petviashvili equation in $\mathbb{R}^{2}$, J. Math. Anal. and Appl., 196 (1995), 566-587.

[4] B. B. Kadmotsev And V. I. Petviashvili, On the stability of solitary waves in weakly dispersive media, Soviet Phys. Dokl., 15 (1970), 539-541.

[5] S. Klainerman And G. Ponce, Global, small amplitude solutions to nonlinear evolution equations, Commun. Pure Appl. Math., 36 (1983), 133-141.

[6] J. C. SAUT, Remarks on the generalized Kadomtsev-Petviashvili equations, Indiana Univ. Math. J., 42 (1993), 1011-1026.

[7] J. C. SAUT, Recent results on the generalized Kadomtsev-Petviashvili equations, Acta Applicandae Mathemticae, 39 (1995), 477-487.

[8] J. SHATAH, Global existence of small solutions to nonlinear evolution equations, J. Differential Equations, 46 (1982), 409-425.

[9] W. A. StRauss, Nonlinear scattering theory at low energy, J. Funct. Anal., 41 (1981), 110-133.

[10] S. K. TURISYN AND G. E. FAL' KOVITCH, Stability of magnetoelastic solutions and self-focusing of sound in antiferromagnet, Soviet Phys. JETP, 62 (1985), 146-152. 